\title{
In-box LOCA accident analysis for the European DEMO water-cooled Reactor
}

\author{
D’Onorio Matteo ${ }^{\mathrm{a}}$, Fabio Giannetti ${ }^{\mathrm{a}}$, Gianfranco Caruso ${ }^{\mathrm{a}}$, Maria Teresa Porfiri ${ }^{\mathrm{b}}$ \\ ${ }^{a}$ Department of Astronautical Electrical and Energy Engineering, Sapienza University of Rome, Rome, Italy \\ ${ }^{b}$ UTFUS -TECN, ENEA CR Frascati, Frascati, Rome, Italy
}

\begin{abstract}
Transient analyses in a water-cooled fusion DEMO (Demonstration Power Plant) reactor have been performed to support the WCLL (Water-Cooled Lithium Lead) breeding blanket design. In this framework, the Design Basis Accident (DBA) analysis of an in-box LOCA has been carried out. The WCLL breeding blanket concept relies on Lithium Lead (LiPb) as breeder, neutron multiplier and tritium carrier, which is cooled by water at $15.5 \mathrm{MPa}$ with an inlet temperature of $295^{\circ} \mathrm{C}$ and an outlet temperature of $328^{\circ} \mathrm{C}$. Water flows in Double-Wall Tubes (DWTs) in order to reduce the probability of water/LiPb chemical interaction. In the case of a LOCA multiple rupture of these tubes is postulated, with consequent leakage of pressurized water in the LiPb side of the module. The analysis has been performed with the MELCOR computer code (ver. 1.8.6) modified for the application in the fusion context. Custom models are employed to simulate the chemical water/ $\mathrm{LiPb}$ interaction in the module. The rupture mass flow rate calculated in water simulation is transformed in its equivalent in terms of hydrogen and unreacted water steam. Both have been treated as non-condensable gas. Two different input decks, one for each fluid considered, have been coupled through an external interface to account for their reciprocal interaction. Pressure and temperature transient behavior in the broken module demonstrate that safety margins are respected during the whole accidental sequence, even though no external safety system is foreseen or actuated. Moreover, attention has paid to the quantity of hydrogen produced in order to support the development of solutions, suitable for DEMO, to prevent hydrogen explosion.
\end{abstract}

Keywords: DEMO; WCLL; In-box LOCA; Fusion reactors; MELCOR; Safety Analysis

\section{Introduction}

Water-Cooled Lithium Lead (WCLL) blanket concept [1] [1] is one of the DEMO (Demonstration Power Plant) blanket concepts candidate for the final design selection. It relies on the use of Lithium-Lead as breeder neutron multiplier and tritium carrier. The breeder is cooled by water, at typical pressurized water reactors (PWR) conditions, flowing in Double-Wall Tubes (DWT), which are used to reduce the probability of water $/ \mathrm{LiPb}$ interaction inside the breeding modules.

However, this probability is not negligible and the rupture of DWTs is included in the list of initiating events for DBA to be studied for the DEMO reactor [3]. This kind of accident has not been yet deeply investigated for the WCLL concept because of the lack of multi-phase safetyrelated system codes able to deal with water and liquid metals. In this context, a preliminary analysis of an in-box LOCA for the WCLL blanket concept has been performed employing custom models. The aim is to evaluate pressure and temperature transients following to the leakage of water from DWTs within one breeder module and the subsequent mass of hydrogen generated during the oxidation reaction between lithium-lead and water.

\section{Relevant design for the LOCA analysis}

For this study, the ENEA WCLL 2015 configuration has been considered [6]. The blanket is divided in 18 Sectors in toroidal direction. Each sector comprises 3 segments in the outboard blanket $(\mathrm{OB})$ and 2 segments in the inboard blanket (IB). Each segment is divided in 7 modules for a total of 378 outboard modules and 252 inboard modules. The equatorial outboard module (OB4) is selected as the affected reference module for the LOCA analysis. It consists of a Eurofer steel box, reinforced by an internal grid of radial-poloidal and poloidal-toroidal plates (stiffeners) to withstand water pressure in case of leakage (Fig. 1).
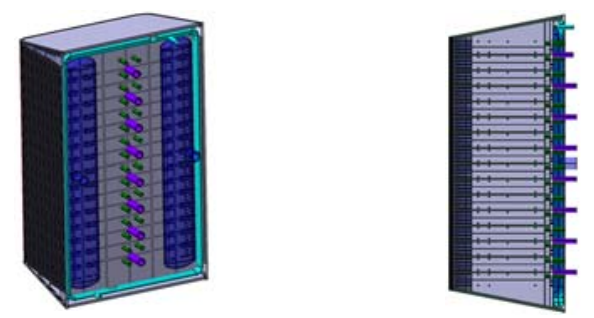

Fig. 1. DEMO Equatorial outboard module

The module box is divided in 16 elementary cells in poloidal direction and 6 channels in toroidal direction. Each elementary cell is divided in the middle by a baffle plate. The PbLi enters from the bottom of the cell, flows in radial-poloidal direction and exits from the top of the elementary cell. First Wall is integrated in the module and cooled by an independent system made of square channels with dimension of $7 \times 7 \mathrm{~mm}$ and pitch of $13.5 \mathrm{~mm}$. BZ cooling tubes are placed along a toroidal-radial direction. The tubes are double walled and have external diameter of $13.5 \mathrm{~mm}$, internal diameter of $8 \mathrm{~mm}$ and thickness of $1.25 \mathrm{~mm}$. Each elementary cell contains 21 tubes. The tubes are grouped and joined to the manifolds of the BZ 
cooling water. The design data for $\mathrm{OB} 4$ are: mass flow rate of $7.38 \mathrm{~kg} / \mathrm{s}$, temperature of $285{ }^{\circ} \mathrm{C}$ and pressure of $15.5 \mathrm{MPa}$ at the blanket inlet, $325^{\circ} \mathrm{C}$ at the blanket outlet. The $\mathrm{LiPb}$ in the breeding modules is at $0.588 \mathrm{MPa}$ and $599 \mathrm{~K}[6]$.

\section{Accident description}

For this in-box LOCA analysis, the complete failure of all tubes in the inversion zone of the central elementary cell of the equatorial module has been considered (Fig. 2). The resulting flow area, postulating a double-ended pipes break, is $2.12 \cdot 10^{-3} \mathrm{~m}^{2}$.

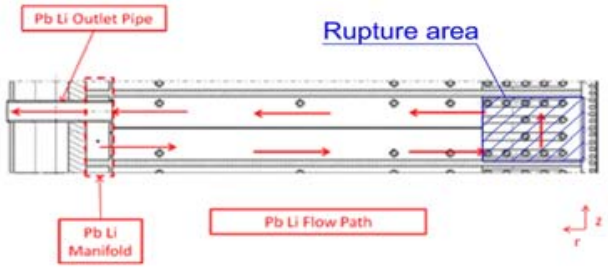

Fig. 2. Flow path of $\mathrm{LiPb}$ in the elementary cells and rupture area considered for the analysis

The rupture is supposed to take place in $5 \mathrm{~s}$ during which the flow area ranges from $0 \%$ to $100 \%$ of the total double break area. As conservative assumption, the affected module is supposed to be in adiabatic conditions respect to other modules and structures. Even if fusion power is terminated by overpressure signal in the breeding blanket module, if the module itself fails, coolant and radioactive inventories could be mobilized towards the Vacuum Vessel. Liquid metal dust/water interaction and radioactivity transport are the main concerns in this scenario.

\section{Methodology and assumptions}

In-box LOCA safety analysis has been performed using the MELCOR ver. 1.8.6 computer code, developed by Sandia National Laboratories for the U.S. Nuclear Regulatory Commission, and modified by Idaho National Laboratory (INL) for the application in the fusion context [4]. The MELCOR code, as other thermal hydraulics system codes, is not able to perform simulations with mixing of two or more different fluids, but only noncondensable gases can be added to the working fluid. The presence of both water and lithium-lead within the breeding blanket therefore cannot be simulated.

To overcome such constraint, two input decks have been detailed with two different working fluids: water and lithium-lead. The two input decks have been coupled through an external Phyton script to account for their reciprocal interaction.

The script performs the following operations:

- An External Data File (EDF-2 in Fig. 3) is created to initialize the MELCOR run for the $\mathrm{H} 2 \mathrm{O}$ case;

- The MELCOR H2O case runs for a timestep; at the end of the run mass flow rate, pressure and other common parameters are written in a new EDF (EDF1 in Fig. 3);

- Such data are used by the Python script to evaluate the amount of hydrogen and heat produced by the chemical reaction between $\mathrm{LiPb}$ and water;
- Such data are added to EDF-1 which is read by MELCOR input for LiPb case;

- The MELCOR LiPb case runs for a timestep; at the end of the run OB4 module pressure and other common parameters are added to the EDF-2 which is used to run the $\mathrm{H} 2 \mathrm{O}$ case for the next time step;

- Iterations are repeated for the other timesteps until the final time is reached.

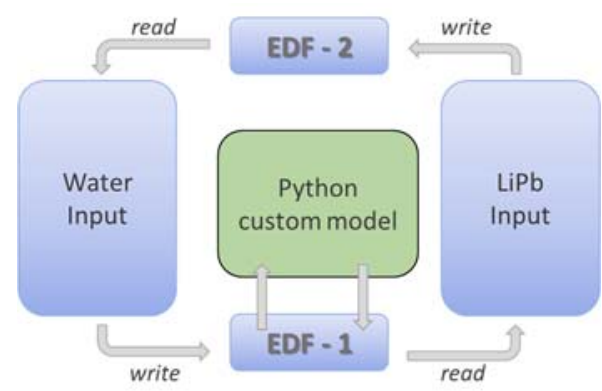

Fig. 3. Calculation methodology scheme

Even adopting this procedure for the analysis, limitations for MELCOR multicomponent capabilities remain. Several simplifications have been made to perform the simulation. To simulate steam injection into the breeding blanket volumes, a user defined non-condensable gas has been used. Pressure and temperature of steam and hydrogen injected are controlled through EDF, assuming an ideal gas isentropic expansion to the pressure of $\mathrm{LiPb}$ side for the evaluation of the steam inlet temperature. The temperature of produced hydrogen is the same of the previous timestep. Then, the energy balance is performed by the code, taking into account the reaction heat, added through an independent heat structure, which heat flux is controlled by the EDF. The hydrogen and heat generated by the LiPb-Water reaction are given by [5]:

$$
\begin{aligned}
& \mathrm{Li}_{17} \mathrm{~Pb}_{83}+8.5 \mathrm{H}_{2} \mathrm{O} \rightarrow 8.5 \mathrm{Li}_{2} \mathrm{O}+8.5 \mathrm{H}_{2}+83 \mathrm{~Pb}+19.0 \frac{\mathrm{kJ}}{\mathrm{mol} \mathrm{LiPb}} \\
& \mathrm{Li}_{17} \mathrm{~Pb}_{83}+17 \mathrm{H}_{2} \mathrm{O} \rightarrow 17 \mathrm{LiOH}+8.5 \mathrm{H}_{2}+83 \mathrm{~Pb}+25.8 \frac{\mathrm{kJ}}{\mathrm{mol} \mathrm{LiPb}}
\end{aligned}
$$

The first one (A) occurs in excess of LiPb, while the second in excess of water. From the thermal and hydrogen generation points of view, the reaction (A) is very conservative.

Preliminary experimental campaigns [7] to estimate the water flow rate in the accident suggested to consider a jet of water penetrating into the $\mathrm{LiPb}$ volume and, as the reaction will occur mainly at the interface between the liquid metal and water, a condition of excess of water has been assumed as more realistic. Therefore, the reaction (B) has been assumed in the present analyses. The reaction of all the water injected into the $\mathrm{LiPb}$ circuit is assumed instantaneous. The reacting water is instantaneously substituted with the correspondent hydrogen generated by the selected chemical reaction. This hypothesis could be very conservative, and in absence of a correct reaction kinetic model implemented, several calculations at different fraction of water participating to the reaction were performed. At this purpose, the C_react parameter has been used to represent the fraction of water that entering the breeding zone reacts with the breeder material. 


\section{MELCOR model}

Water circuit has been nodalized using 34 control volumes (CVHs) connected with flow path to model all reactor components. The 17 undamaged sectors have been modeled using one $\mathrm{CVH}$. The damaged sector has been modeled in detail as reported in Fig. 4. Water circuit is supposed to break in the central part of the OB4 module and the break involves all the 21 DWTs. The broken module (OB4 in Fig. 4) is connected to a time dependent volume ( $\mathrm{LiPb}$ TDV volume in Fig. 4) to simulate the $\mathrm{LiPb}$ side. This volume receives information during the MELCOR $\mathrm{LiPb}$ run to set the right pressure and temperature for each time step.

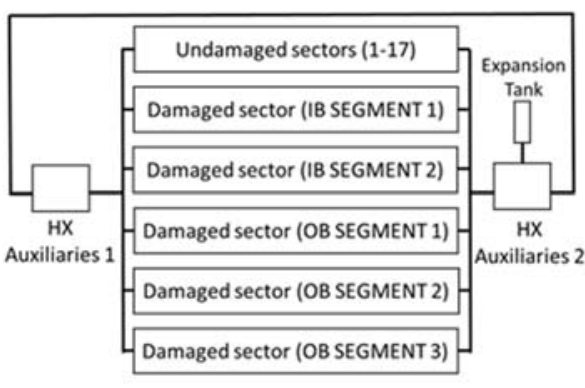

H2O sector 18 (OB SEGMENT 3)

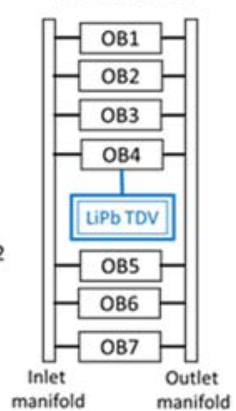

Fig. 4. Water circuit scheme

The lithium lead circuit has been nodalized with 23 control volumes (CVHs). Two different time dependent control volumes (the blue in Fig. 5) have been used to simulate the effects of LiPb-water reaction within the OB4 module. Their function is to inject steam and hydrogen in the breeding zone depending on the fraction of reacting water.

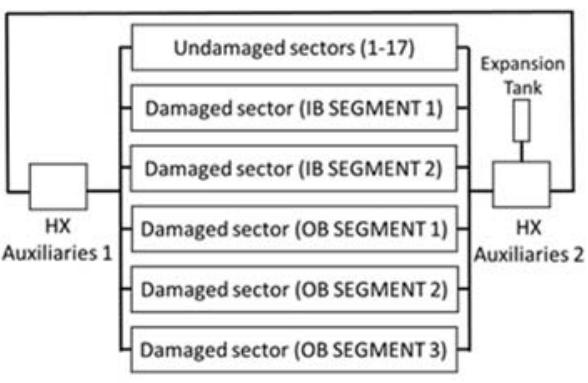

Fig. 5. Lithium lead circuit scheme

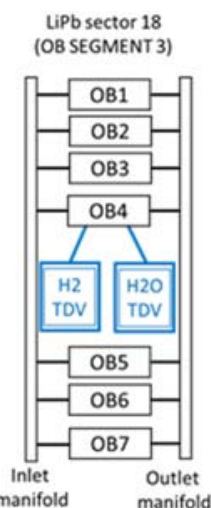

For both circuits, ex-vessel components including loop distributor, steam generator, pumps and pressurizer have been modeled using 2 CVHs. BZ cooling loops, manifolds, caps and back supporting structure (BSS) have been modeled through Heat structures (HSs). The decay heat has been assumed as $1.2 \%$ of the full power.

\section{Results and discussion}

Both $\mathrm{LiPb}$ and water runs exchange information on parameters thanks to the custom interface, which made possible to perform coupled analyses. Simulations time has been selected to reach thermal and mechanical equilibrium between the two loops.
Water mass flow rate from the rupture transient (

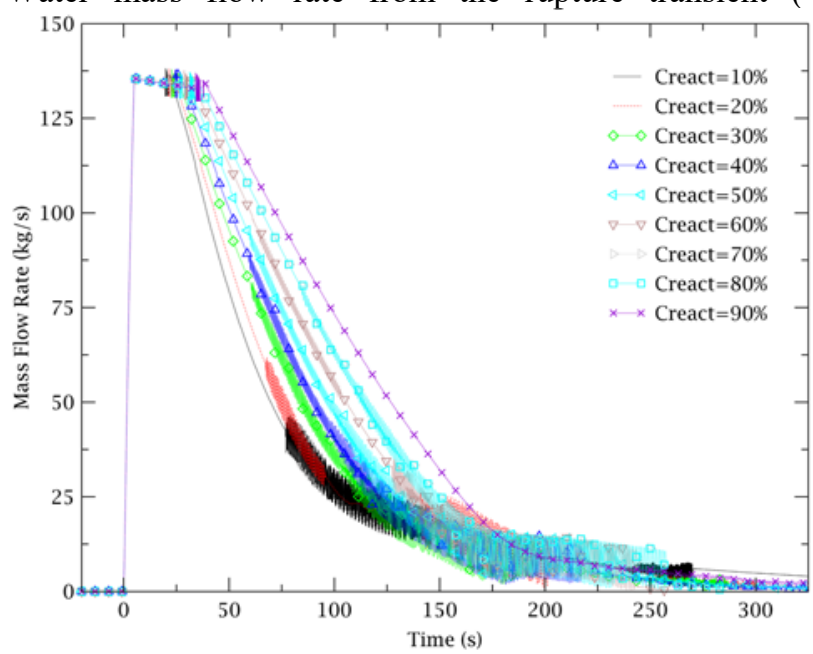

Fig. 6. Water mass flow rate from rupture

) is characterized by an initial rapid increase from 0 to $5 \mathrm{~s}$ when the maximum of about $136 \mathrm{~kg} / \mathrm{s}$ is achieved. After that, the mass flow rate decreases slowly until $25 \mathrm{~s}$ and then declines until the end of the transient. The intermediate and final phases are characterized by instabilities due to reduced pressure difference and vaporization effects within water circuit.

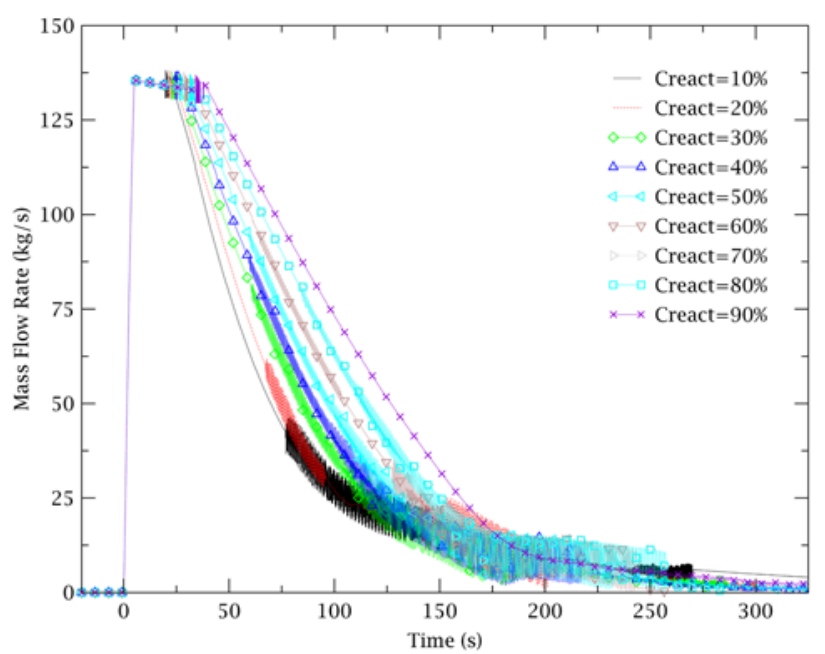

Fig. 6. Water mass flow rate from rupture

Non-reacting water mass flow rate entering the breeding zone for different values of $C_{\text {react }}$ is reported in

Fig. 7. Non-reacting water entering in the breeding module for different reaction coefficients

. The larger is the reaction rate, the lower is the nonreacting water flowing from the water side to the breeding zone. At the end of the transient, with a $C_{\text {react }}$ of $10 \%$, about 9.5 ton of water flowed from the DWTs to the breeding zone. 


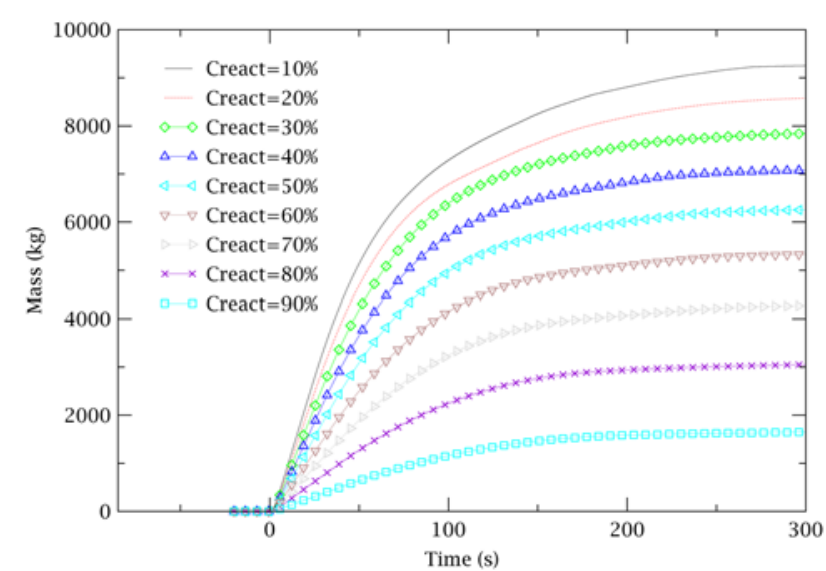

Fig. 7. Non-reacting water entering in the breeding module for different reaction coefficients

Hydrogen production rate (Errore. L'origine riferimento non è stata trovata.) was obtained using the reaction with excess of water. For each kilogram of reacted water $0.056 \mathrm{~kg}$ of hydrogen are produced. The total amount of hydrogen in the broken module reaches a maximum of about $103 \mathrm{~kg}$ after $225 \mathrm{~s}$ for a $C_{\text {react }}$ of $90 \%$. The fast release of these amounts of water and hydrogen within the module, along with the reaction energy release, lead to a rapid pressurization of the $\mathrm{LiPb}$ loop.

Pressure in the module (Errore. L'origine riferimento non è stata trovata.) is $3.6 \mathrm{MPa}$ at $5 \mathrm{~s}$ after the break. After the initial peak, pressure in the damaged module drops to a value of $2.9 \mathrm{MPa}$ and then it starts to increase again due to the combined effect of mass release and reaction. The maximum value of pressure is $14.99 \mathrm{MPa}$ and could be reached between $125 \mathrm{~s}$ and $220 \mathrm{~s}$ depending on the $C_{\text {react }}$ parameter.

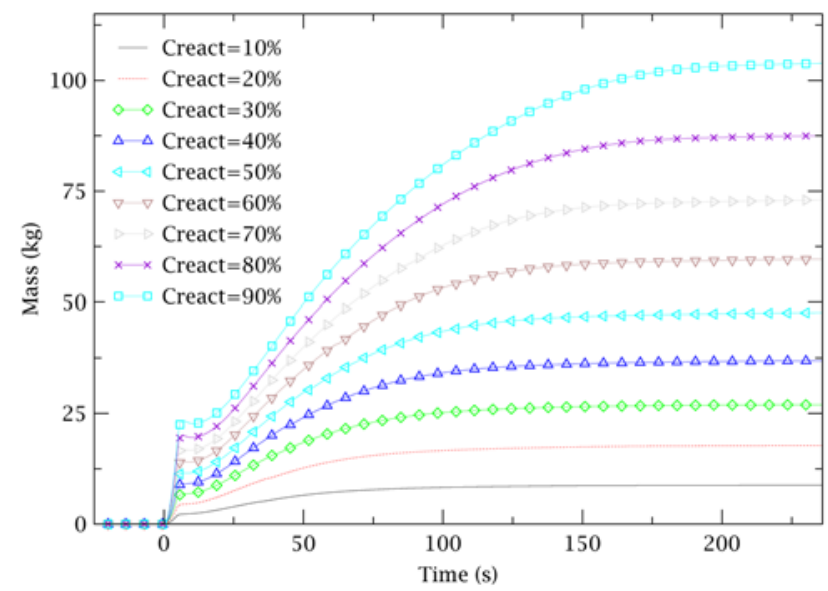

Fig. 8. Hydrogen generation rate for different reaction coefficients

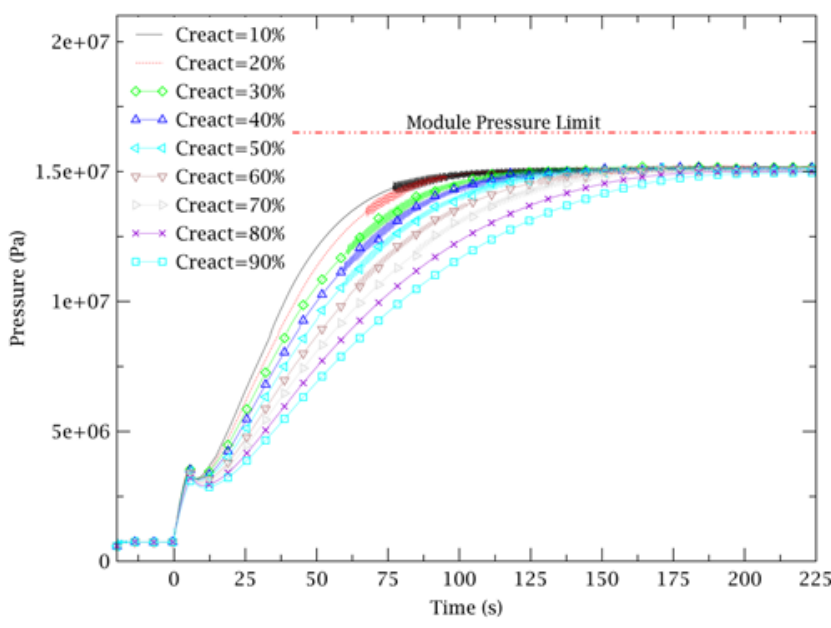

Fig. 9. Breeding module pressure for different reaction coefficients

The temperature in the damaged breeder module increases because of heat released by the reaction and decay heat produced after reactor shutdown (Errore. L'origine riferimento non è stata trovata.). The maximum temperature of $627 \mathrm{~K}$ is reached by the module for a $C_{\text {react }}=90 \%$. This temperature is below the limit temperature value of Eurofer $(823 \mathrm{~K})$.

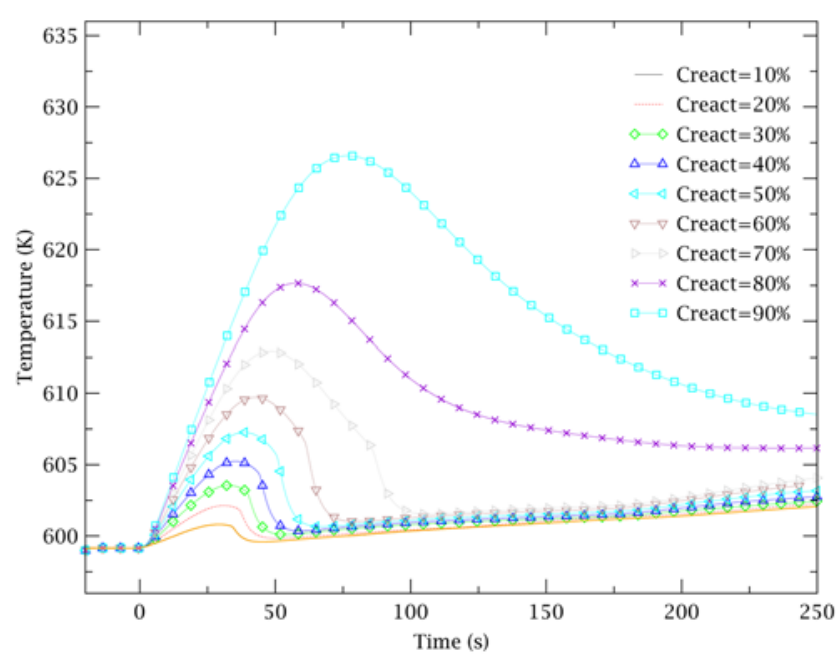

Fig. 10. Breeding module temperature for different reaction coefficients

\section{Conclusions}

The work presented here describes an application of a custom methodology developed to analyze the effects of an in-box LOCA for the WCLL blanket concept of the EU-DEMO reactor. Due to the difference in the operating pressure, after the tubes rupture, water enters the breeding module and reacts with lithium lead generating hydrogen and thermal power. To better simulate the mutual influence of the two circuits during the transient, a python script has been developed and used to couple the two sides, which uses two working fluids and requires different inputs. The results obtained for LiPb loop show first a fast pressurization of breeding blanket in which pressure reaches the maximum value of $14.9 \mathrm{MPa}$ which is below the design pressure limit of breeding blanket box which are verified to withstand a pressure of $15.5 \mathrm{MPa}+$ $10 \%$ [6]. The parametric analysis, carried out using the 
fraction of reacting water as parameter, shows faster pressurization for smaller reaction coefficients. This result is related to the methodology used for simulations. The rupture mass flow rate calculated in water simulation is transformed in its equivalent in terms of hydrogen and unreacted water vapor. Both the reaction products are treated as non-condensable gas in $\mathrm{LiPb}$ simulation. The higher amount of steam for lower reaction coefficient leads to faster pressurization, as it does not condense.

The developed model will be improved to take into account all these effects, in order to better analyze and understand the reliability of the results.

\section{Acknowledgements}

This work has been carried out within the framework of the EUROfusion Consortium and has received funding from the Euratom research and training programme 20142018 under grant agreement No.633053. The views and opinions expressed herein do not necessarily reflect those of the European Commission

\section{References}

[1] G. Federici et al., DEMO design activity in Europe: Progress and updates, Fusion Eng. Des., 2019, https://doi.org/10.1016/j.fusengdes.2018.04.001, in press.

[2] A. Tassone, et al., Recent progress in the WCLL breeding blanket design for the DEMO fusion reactor, IEEE Trans. Plasma Sci. 46 (2018) 1446-1457.

[3] T. Pinna, Identification of accident sequences for the DEMO plant, Fusion Eng. Des. 124 (2017) 1277-1280.

[4] B.J. Merrill, A recent version of MELCOR for fusion safety applications, Fusion Eng. Des. 85 (2010) 14791483.

[5] K.A. McCarthy, Safety issues related to liquid metals, APEX meeting, Albuquerque (USA), July 1998.

[6] A. Del Nevo, et al., WCLL breeding blanket design and integration for DEMO 2015: status and perspectives, Fusion Eng. Des. 124 (2017) 682-686.

[7] Ciampichetti, I. Ricapito, A. Aiello, G. Benamati: water large leaks into liquid $\mathrm{Pb}-17 \mathrm{Li}$ : first experimental results on LIFUS 5 facility, Fusion Eng. Des. 69 (2003) 563-567. 